\title{
Os impactos da inserção feminina no mercado de trabalho sobre o perfil nutricional e os hábitos alimentares de escolares adolescentes
}

The impacts of female insertion in the labor market on the nutritional profile and the eating habits

\author{
of adolescent students
}

Los impactos de la inserción femenina en el mercado laboral sobre el perfil nutricional y los hábitos alimentarios de estudiantes adolescentes

Recebido: 27/05/2021 | Revisado: 02/06/2021 | Aceito: 06/06/2021 | Publicado: 20/06/2021

\author{
Rafael Veras Castelo Branco \\ ORCID: https://orcid.org/0000-0003-2100-1179 \\ Universidade Federal do Ceará, Brasil \\ E-mail: rafaelverass@alu.ufc.br \\ Adriana Camurça Pontes Siqueira \\ ORCID: https://orcid.org/0000-0002-2131-7598 \\ Universidade Federal do Ceará, Brasil \\ E-mail: adriana.siqueira@ufc.br \\ Diana Valesca Carvalho \\ ORCID: https://orcid.org/0000-0001-5355-2309 \\ Universidade Federal do Ceará, Brasil \\ E-mail: dianacarvalho@ufc.br \\ Elizangela Alves de Oliveira \\ ORCID: https://orcid.org/0000-0002-0983-9254 \\ Universidade Federal do Ceará, Brasil \\ E-mail: elizangela@alu.ufc.br \\ Lucas Vasconcelos de Oliveira \\ ORCID: https://orcid.org/0000-0002-9508-6972 \\ Universidade Federal do Ceará, Brasil \\ E-mail: lucasvascdeoliveira@alu.ufc.br \\ Dhébora Paiva Lima \\ ORCID: https://orcid.org/0000-0002-0645-0200 \\ Universidade Federal do Ceará, Brasil \\ E-mail: dheborapaiva@alu.ufc.br
}

\begin{abstract}
Resumo
Inúmeros fatores justificam o aumento do número de casos de sobrepeso e obesidade entre a população brasileira nas últimas décadas, entre eles a inserção da mulher no mercado de trabalho, realidade que altera a estrutura familiar e os modelos de alimentação, sobretudo, de crianças e adolescentes. A presente pesquisa objetiva avaliar os impactos da inserção feminina no mercado de trabalho sobre o estado nutricional de escolares adolescentes, por meio da análise dos hábitos alimentares e do perfil antropométrico de 67 estudantes do $1^{\circ}$ ao $3^{\circ}$ ano do ensino médio, de uma escola pública de tempo integral no município de Fortaleza/CE. Essa avaliação foi viabilizada pela aplicação de questionário validado junto aos alunos e pela leitura de seus dados antropométricos, utilizando como parâmetro os gráficos de referência das curvas de crescimento da Organização Mundial de Saúde (OMS), onde esses valores foram correlacionados com a idade e o sexo dos voluntários e convertidos em escores-z. Assim, pode-se perceber que $53,8 \%$ dos estudantes com sobrepeso têm suas mães inseridas no mercado de trabalho, $80 \%$ dos alunos obesos aparecem entre o grupo de famílias sustentadas pela mãe e 50\% dos que receberam diagnóstico para obesidade grave estão entre os adolescentes providos pela figura materna. Dessa forma, pondera-se que a mulher ainda desempenha o papel de cuidado no contexto familiar e que se faz urgente a implementação de ações de Educação Alimentar e Nutricional nas escolas no sentido de promover, entre os alunos, práticas alimentares saudáveis por meio do desenvolvimento de sua autonomia.
\end{abstract}

Palavras-chave: Gastronomia; Saúde; Alimentação; Família; Escola.

\section{Abstract}

Innumerable factors justify the increase in the number of cases of overweight and obesity among the Brazilian population in the latest decades, including the insertion of women in the labor market, a reality that alters the family structure and the nutrition models, mostly for children and adolescents. The present research aims to evaluate the impacts of female insertion in the labor market on the nutritional status of adolescent students, analyzing the 
anthropometric profile and the eating habits of 67 high-school students from the 1st to the 3rd grade of a full-time public school from the city of Fortaleza/CE. This evaluation was made possible by the application of a validated questionnaire with the students and by reading their anthropometric data, based on the World Health Organization (WHO) growth curves reference charts, in which these values were correlated with age and gender of the volunteers and converted into z-scores. Therefore, it can be seen that $53.8 \%$ of students diagnosed with overweight have their mothers in the labor market, $80 \%$ of the obese students appear among the group of families supported by the mother and $50 \%$ of those who have been diagnosed with severe obesity are among the adolescents provided by the maternal figure. Thus, it is considered that women still play the role of caregiving in the family context and that it is urgent to implement actions of Food and Nutrition Education in schools in the sense of promoting healthy eating practices among students by means of their autonomy development.

Keywords: Gastronomy; Health; Food; Family; School.

\section{Resumen}

Innúmeros factores justifican el aumento en el número de casos de sobrepeso y obesidad entre la población brasileña en las últimas décadas, entre ellos, la inserción de las mujeres en el mercado laboral, realidad que altera la estructura familiar y los modelos de alimentación, especialmente los niños y adolescentes. La presente investigación tiene como objetivo evaluar los impactos de la inserción femenina en el mercado laboral sobre el estado nutricional de los estudiantes adolescentes, a través del análisis de los hábitos alimentarios y el perfil antropométrico de 67 estudiantes de $1^{\circ}$ a $3^{\circ}$ año de la secundaria, de una escuela pública de tiempo completo en la ciudad de Fortaleza/CE. Esta evaluación fue posible mediante la aplicación de un cuestionario validado con los estudiantes y la lectura de sus datos antropométricos, utilizando como patrones las tablas de referencia de las curvas de crecimiento de la Organización Mundial de la Salud (OMS), donde estos valores se correlacionan con la edad y el sexo de los voluntarios y son convertidos en puntajes z. Así, se percibe que el 53,8\% de los estudiantes diagnosticados con sobrepeso tienen a sus madres en el mercado laboral, el $80 \%$ de los estudiantes obesos aparecen entre el grupo de familias sustentado por la madre y el $50 \%$ de los que fueron diagnosticados con obesidad severa se encuentran entre los adolescentes proveídos por la figura materna. De esa manera, se considera que las mujeres aún desempeñan el papel del cuidado en el contexto familiar y que es urgente implementar acciones de Educación Alimentaria y Nutricional en las escuelas con el fin de promover prácticas de alimentación saludable entre los estudiantes a través del desarrollo de su autonomía.

Palabras clave: Gastronomía; Salud; Alimentación; Familia; Escuela.

\section{Introdução}

O Brasil vivencia, desde os anos de 1970, um fenômeno denominado por especialistas e pesquisadores como transição nutricional, que, segundo Santos et al. (2019, p. 479), consiste em "um processo de modificações sequenciais no padrão de nutrição e consumo, que acompanha mudanças econômicas, sociais, demográficas e de perfil de saúde das populações”. No contexto brasileiro, portanto, este fato diz respeito à redução da prevalência dos déficits nutricionais e ao concomitante aumento do número de casos de sobrepeso e obesidade entre a população do país. O sobrepeso e a obesidade, no entanto, não são problemas isolados, visto que podem acarretar diversas doenças crônicas, como a hipertensão arterial, a hipercolesterolemia, o diabetes mellitus, as doenças cardiovasculares e alguns tipos de câncer (Souza, 2010).

Palco de fome e desnutrição epidêmica em suas regiões Norte e Nordeste nos anos de 1950, o Brasil consegue, a partir da década de 70, registrar a diminuição da prevalência de desnutrição entre crianças de até 5 anos e, consequentemente, do retardo de estatura. Esses números são, todavia, mais substanciais entre a população da zona urbana e, a partir de 1989, mais evidentes nas regiões Norte e Nordeste do país. Ao todo, entre 1970 e os anos 2000, o Brasil assistiu a uma diminuição de cerca de $72 \%$ dos índices de deficiência nutricional, contudo, assimilou um já referido grande problema, ponto-chave da transição nutricional brasileira, a prevalência de sobrepeso e obesidade (Batista Filho \& Rissin, 2003).

De acordo com a Pesquisa Nacional de Saúde 2019 (PNS 2019), entre os brasileiros com idade acima de 18 anos, mais da metade, cerca de 96 milhões, apresentam excesso de peso. Entre os adolescentes com idade entre 15 e 17 anos, os dados também são alarmantes, tendo sido diagnosticados com sobrepeso 1,8 milhão de pessoas nessa faixa etária, onde o público feminino destaca-se tanto para o sobrepeso como para a obesidade (Brasil, 2020). Tal situação já se mostrava preocupante desde 2016. Segundo o relatório divulgado pelo Sistema de Vigilância Alimentar e Nutricional (SISVAN) daquele 
ano, quase um quarto dos adolescentes brasileiros apresentaram Índice de Massa Corpórea (IMC) acima do recomendado. Por outro lado, o relatório apontou dados positivos em relação aos índices de magreza e magreza extrema (Brasil, 2016b).

Embora se considere bastante positiva a superação da desnutrição, é crítico o nível de obesidade entre os adolescentes brasileiros, já que esta é considerada, pela Organização Mundial de Saúde (OMS), uma epidemia global e um grave problema de saúde pública. Além de problemas de saúde física, a obesidade acarreta em adolescentes problemas psicológicos, como a baixa autoestima, que se reflete em suas relações interpessoais e no seu rendimento na vida social e no ambiente escolar. Essa situação acende um importante alerta à medida que a infância e a adolescência são fases cruciais no processo de educação alimentar e formação dos hábitos alimentares da população (Santos et al., 2019).

Inúmeros fatores podem justificar essa mudança de cenário, entre eles a transição demográfica e territorial ocorrida a partir de 1950, em que a população brasileira deixa de ser, em sua maioria rural e passa a ser hegemonicamente urbana; e a inserção da mulher no mercado de trabalho, com consequente alteração na estrutura familiar e visível transformação na qualidade e nos modelos de alimentação, em que se observa o aumento do consumo de produtos industrializados, prontos, ou fora do lar - a exemplo dos fast-foods - em detrimento de refeições preparadas em casa (Batista Filho \& Rissin, 2003; Souza, 2010). Lelis et al. (2012) apontam que a figura feminina acumula responsabilidade sobre $80 \%$ das atividades alimentares domésticas, o que, no atual contexto, soma-se às obrigações laborais, gerando sobrecarga, visto que não há mecanismos de suporte social a elas nesse sentido. Assim, a alimentação fora dos domicílios deixa de ser esporádica e passa a fazer parte da rotina de muitas famílias brasileiras.

Considerando isso, a presente pesquisa objetiva avaliar os impactos da inserção feminina no mercado de trabalho sobre a situação nutricional e os hábitos alimentares de escolares adolescentes, por meio da análise da alimentação e do perfil antropométrico de estudantes de ensino médio, com idade entre 15 e 18 anos, de uma escola pública de tempo integral no município de Fortaleza/CE. É também objetivo deste trabalho discutir soluções para o problema a partir da concepção do papel do poder público, da sociedade civil e das próprias famílias no processo de educação alimentar e nutricional de crianças e adolescentes.

\section{Metodologia}

A presente pesquisa foi desenvolvida a partir de quatro principais etapas: 1- A construção da situação-problema, através da leitura do perfil nutricional da população brasileira, com foco em escolares adolescentes, tendo como referência a Pesquisa Nacional de Saúde 2019 (PNS 2019) e a Pesquisa Nacional de Saúde do Escolar 2015 (PeNSE 2015); 2 - O levantamento dos dados antropométricos e dos hábitos alimentares de escolares adolescentes, tendo como recorte 67 estudantes do $1^{\circ}$ ao $3^{\circ}$ ano do ensino médio de uma escola de tempo integral da rede pública do município de Fortaleza/CE; 3 - O diagnóstico da situação nutricional e dos hábitos alimentares dos alunos que se voluntariaram à pesquisa; 4 - A análise da correlação entre o perfil nutricional e os hábitos alimentares desses adolescentes com o fato de a figura feminina de suas famílias, aqui representada pela mãe, estar ou não inserida no mercado de trabalho.

A construção da situação-problema parte da avaliação da PNS 2019 e da PeNSE 2015, somada à revisão de literatura realizada entre os meses de março de 2019 e março de 2021, abrangendo artigos científicos relacionados à transição nutricional observada no Brasil, ao perfil antropométrico de brasileiros e brasileiras, aos fatores vinculados à incidência de sobrepeso e obesidade entre esta população, com foco em escolares adolescentes, bem como aos impactos da inserção da mulher no mercado de trabalho sobre o modelo de alimentação das famílias brasileiras. Para tanto, foram realizadas buscas de pesquisas/artigos no portal de periódicos da CAPES e nas bases de dados Scielo e Google Scholar, utilizando os seguintes descritivos: "transição nutricional", "déficit estatural", "sobrepeso", "obesidade", "adolescentes", "Brasil”, "IMC”, "mulher", "mercado de trabalho". 
Para a revisão de literatura, foram priorizados os artigos elaborados a partir do ano de 2010 e que trouxessem uma análise consistente sobre a mudança do perfil nutricional da população brasileira nos últimos 50 anos e sobre os fatores que podem estar relacionados a essa alteração de cenário. A PNS 2019 e a PeNSE 2015 foram fontes para atualização de dados relacionados à situação nutricional do povo brasileiro, servindo como parâmetro de desenvolvimento desta pesquisa no sentido de que traçam a realidade do país.

Dessa forma, o estudo aqui desenvolvido tem cunho exploratório, considerando que toda pesquisa científica exige uma imersão bibliográfica e pressupõe uma consistente revisão de literatura, sobretudo, como meio de justificar a sua viabilidade e relevância, mas também como forma de reunir e disponibilizar o máximo de informações sobre o assunto. Além disso, a investigação assume uma abordagem qualitativa por contemplar observações e interpretações dos pesquisadores acerca do fenômeno avaliado a partir da seleção e análise de outras pesquisas e produções acadêmicas, mas também adota uma metodologia quantitativa e transversal, visto que o levantamento dos dados antropométricos e dos hábitos alimentares dos voluntários ocorreu através da aplicação de questionário validado (Rodrigues, 2013), em que constam interrogações objetivas quanto ao peso e altura dos alunos envolvidos, alimentos que eles costumam consumir e em qual frequência, além dos aspectos socioeconômicos de suas famílias, possibilitando a construção de um panorama da situação-problema e sua correlação por meio de análise estatística com aplicação de porcentagem (Andrade, 2010; Lüdke \& André, 1986; Pereira et al., 2018).

Destaca-se que a aplicação do questionário foi realizada após a assinatura dos Termos de Assentimento (TA) e dos Termos de Consentimento Livre e Esclarecido (TCLE) pelos alunos e seus responsáveis, e com auxílio de pesquisadores, que também estavam encarregados pela aferição dos dados antropométricos e posterior cálculo do Índice de Massa Corpórea (IMC), assim como pela tabulação e análise estatística desses dados em planilha do Excel®.

A aferição de peso e altura foi realizada no mesmo local da aplicação do questionário, nas salas de aula da escola, por pesquisadores previamente treinados, utilizando estadiômetro portátil padrão e balança digital da marca CAMRY® com capacidade para $150 \mathrm{~kg}$ e sensibilidade de $100 \mathrm{~g}$, instalada em local afastado da parede, com superfície plana, firme e lisa. Durante o procedimento, foi solicitado que os voluntários ficassem descalços, com os pés unidos, em posição ereta, olhando para frente. O diagnóstico nutricional, por conseguinte, foi realizado tomando como referência os dados de IMC e sua correlação com idade e sexo dos participantes, sendo estes valores convertidos em escores-z, a partir dos pontos de corte recomendados pela OMS, através de seus gráficos de referência das curvas de crescimento - critério IMC/Idade -, que também são adotados pelo Ministério da Saúde (World Health Organization, 2007; Ministério da Saúde, 2019).

De acordo com o Laboratório de Avaliação Nutricional das Populações (LANPOP) (2020) da Universidade de São Paulo - USP, os escores-z são "estimadores que quantificam a distância de um valor observado em relação à mediana de uma população", ou seja, compara os dados de um indivíduo com os dados referentes a um grupo maior. No caso da avaliação do estado nutricional de crianças e adolescentes com idade entre 5 e 19 anos, confronta os dados dos indivíduos em questão com valores obtidos a partir da avaliação de populações consideradas sadias e representadas em um gráfico. Segundo Nota Técnica do Sistema de Vigilância Alimentar e Nutricional (SISVAN) (2019), "estes gráficos correspondem a curvas que refletem a distribuição desses indicadores em uma população de referência, isto é, aquela que inclui dados referentes a indivíduos sadios, vivendo em condições socioeconômicas, culturais e ambientais satisfatórias".

Compreendido isso, para o cálculo do IMC, foi utilizada a seguinte fórmula: peso atual em quilogramas dividido pelo quadrado da altura em metros - peso(kg)/estatura $(\mathrm{m})^{2}$-, sendo os valores obtidos a partir desta fórmula, como já destacado, correlacionados com a idade e o sexo dos voluntários e, posteriormente, convertidos em escores-z, em que escore-z $<-3$ representa diagnóstico para magreza acentuada; $\geq-3$ e $<-2$, magreza; $\geq-2$ e $\leq+1$, eutrofia; $\geq+1$ e $\leq+2$, sobrepeso; $\geq+2$ e $\leq$ +3 , obesidade; e $>+3$, obesidade grave. (World Health Organization, 2007). 
Os gráficos de referência com as curvas de crescimento da OMS para meninas e meninos com idade entre 5 e 19 anos estão apresentados nas Figuras 1 e 2, respectivamente.

Figura 1: Gráfico da curva de crescimento da OMS para meninas com idade entre 5 e 19 anos - critério IMC/Idade.

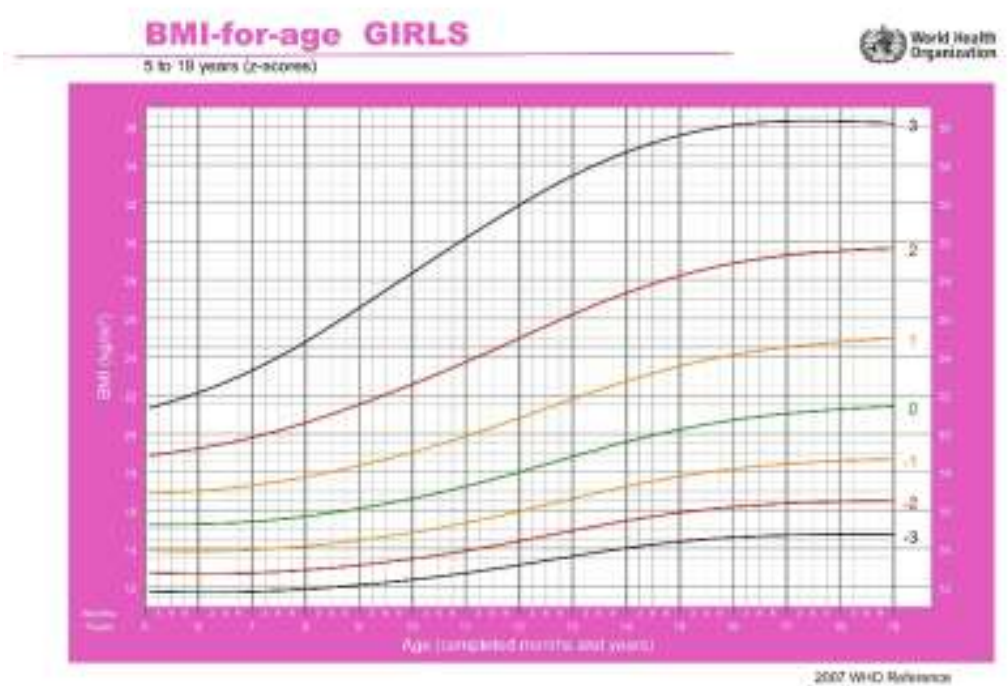

Fonte: World Health Organization (2007).

Figura 2: Gráfico da curva de crescimento da OMS para meninos com idade entre 5 e 19 anos - critério IMC/Idade.

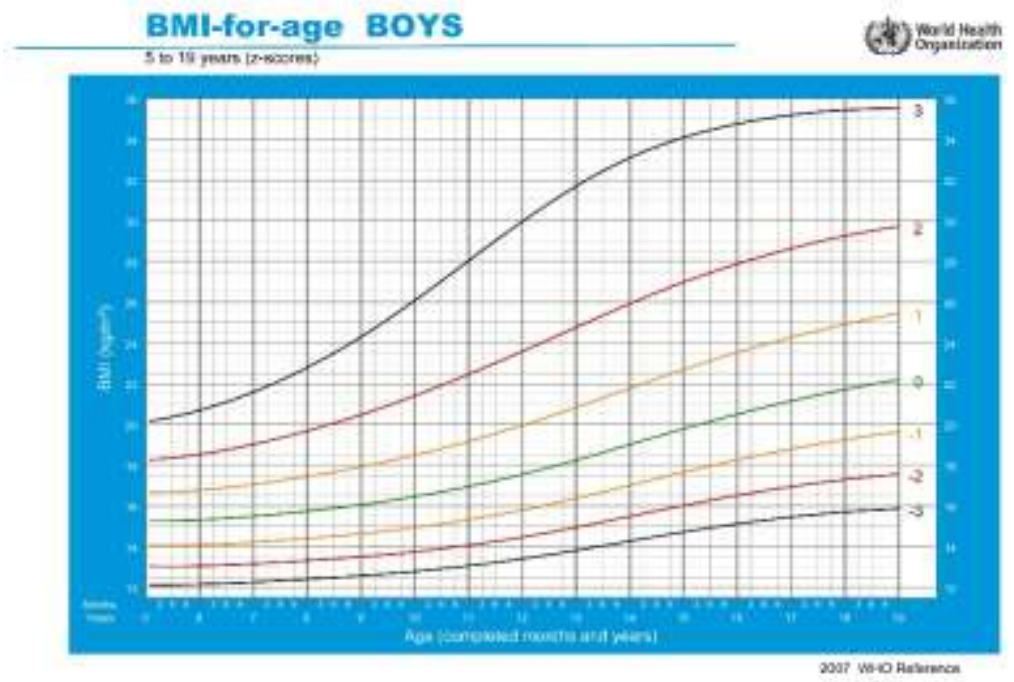

Fonte: World Health Organization (2007).

Já a avaliação dos hábitos alimentares dos alunos voluntários, viabilizada pela aplicação de questionário validado, analisou a realização diária das refeições consideradas padrões na rotina das famílias brasileiras (café da manhã, almoço e jantar), bem como a frequência do consumo alimentar fora de casa, em fast-foods. Também são apontadas algumas das escolhas alimentares dos envolvidos na pesquisa, tomando como referência a categorização de alimentos definida pelo Guia Alimentar para a População Brasileira, em que se considera "o tipo de processamento empregado na sua produção" (Brasil, 2014). Entende-se, portanto, como alimentos in natura ou minimamente processados aqueles obtidos diretamente da natureza, seja de origem vegetal ou animal, e que não tenham sofrido alterações em sua forma natural, ou que tenham passado por alterações mínimas; óleos, gorduras, açúcares e sal, aqueles extraídos de alimentos in natura e que são utilizados como 
tempero; alimentos processados, os fabricados e conservados a partir da adição de sal e açúcar; e alimentos ultraprocessados, aqueles que passam por técnicas e adição de ingredientes de uso exclusivamente industrial (Brasil, 2014).

Finalmente, os dados obtidos quanto ao estado nutricional e aos hábitos alimentares dos alunos envolvidos na pesquisa foram correlacionados com a atuação ou não da figura materna e/ou responsável feminina fora de casa, com o objetivo de compreender e analisar o impacto da inserção da mulher no mercado de trabalho sobre a rotina alimentar e o perfil nutricional de escolares adolescentes. Para identificação da atuação da figura feminina no mercado de trabalho, os alunos foram interrogados, através do questionário, sobre quem chefiava suas famílias, considerando-se, assim, as famílias chefiadas pela mãe ou pela mãe e pelo pai, aquelas em que a matriarca está em plena atividade laboral.

\section{Resultados e Discussão}

A partir da análise dos dados antropométricos, do cálculo do IMC e de sua correlação com a idade e o sexo dos alunos envolvidos nesta pesquisa por meio dos gráficos de referência das curvas de crescimento da OMS, constatou-se que, dentre os 67 estudantes, a maioria (68,66\%) apresentou quadro de eutrofia, ou seja, um bom estado nutricional. No entanto, 19,41\% foram diagnosticados com sobrepeso, 7,46\% com obesidade, 2,98\% com obesidade grave e 1,49\% com magreza (Figura 3). O perfil nutricional dos avaliados reflete, portanto, uma realidade nacional. De acordo com dados divulgados pelo Instituto Brasileiro de Geografia e Estatística (IBGE), através da PNS 2019, 19,4\% de todos os adolescentes brasileiros com idade entre 15 e 17 anos apresentam prevalência de sobrepeso, e 6,7\% de obesidade (Brasil, 2020). Pontua-se, no entanto, que o índice de obesidade entre os voluntários da presente pesquisa está acima da média nacional e dos números obtidos por Guimarães et al. (2020) em avaliação realizada com 33 adolescentes matriculados em escolas públicas do município de Teresina, no Estado do Piauí, onde apenas 3\% deles foram diagnosticados com obesidade.

Figura 3: Estado nutricional dos escolares adolescentes no município de Fortaleza, CE.

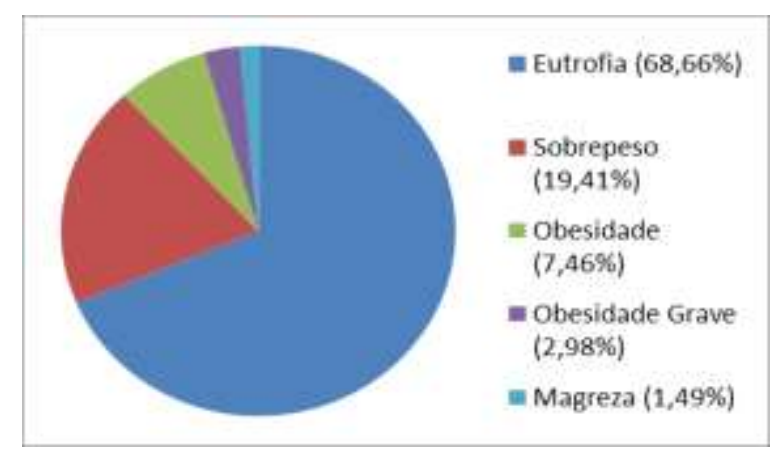

Fonte: Autores

Os resultados encontrados no presente estudo e apresentados na Figura 3, acima, também se aproximam dos números divulgados pela PeNSE 2015, em que 23,7\% dos estudantes brasileiros com idade entre 13 e 17 anos foram diagnosticados com peso acima do considerado desejável, enquanto que 7,8\% encontravam-se no estágio de obesidade (Brasil, 2016a). Para a análise do perfil antropométrico, tanto a PNS 2019 quanto a PeNSE 2015 seguiram as mesmas recomendações da Organização Mundial de Saúde postas nesta pesquisa, com a utilização do Índice de Massa Corpórea, levando em consideração também sexo e idade para diagnósticos de prevalência de baixo peso, excesso de peso e obesidade entre os envolvidos.

\subsection{Estado nutricional de escolares adolescentes e sua correlação com a inserção feminina no mercado de trabalho}

Quando avaliada a relação dos casos de sobrepeso, obesidade e obesidade grave entre os alunos voluntários desta pesquisa com o fato de a figura feminina de seus lares estar em plena atividade laboral, chegou-se aos seguintes resultados: 
53,8\% dos estudantes diagnosticados com sobrepeso têm suas mães inseridas no mercado de trabalho, cumprindo o papel de chefe de família, enquanto que 38,5\% tem recursos financeiros advindos do pai e 7,7\% de outros; quando o diagnóstico se refere à obesidade, $80 \%$ dos alunos encaixados neste perfil nutricional aparecem entre o grupo de famílias sustentadas pela mãe e apenas $20 \%$ por outros; nos quadros de obesidade grave, $50 \%$ deles aparecem entre alunos providos pela mãe e $50 \%$ por outros (Figura 4). Nota-se, dessa forma, que, nas famílias chefiadas pela mãe e que contam com a ausência da mulher na rotina do ambiente doméstico, acontece a maior incidência dos casos de sobrepeso e, sobretudo, obesidade. Não foram detectados adolescentes obesos ou com obesidade grave entre as famílias chefiadas pelo pai.

Figura 4: Relação estado nutricional de escolares adolescentes x Inserção da mulher no mercado de trabalho (famílias chefiadas pela mãe) no município de Fortaleza, CE.

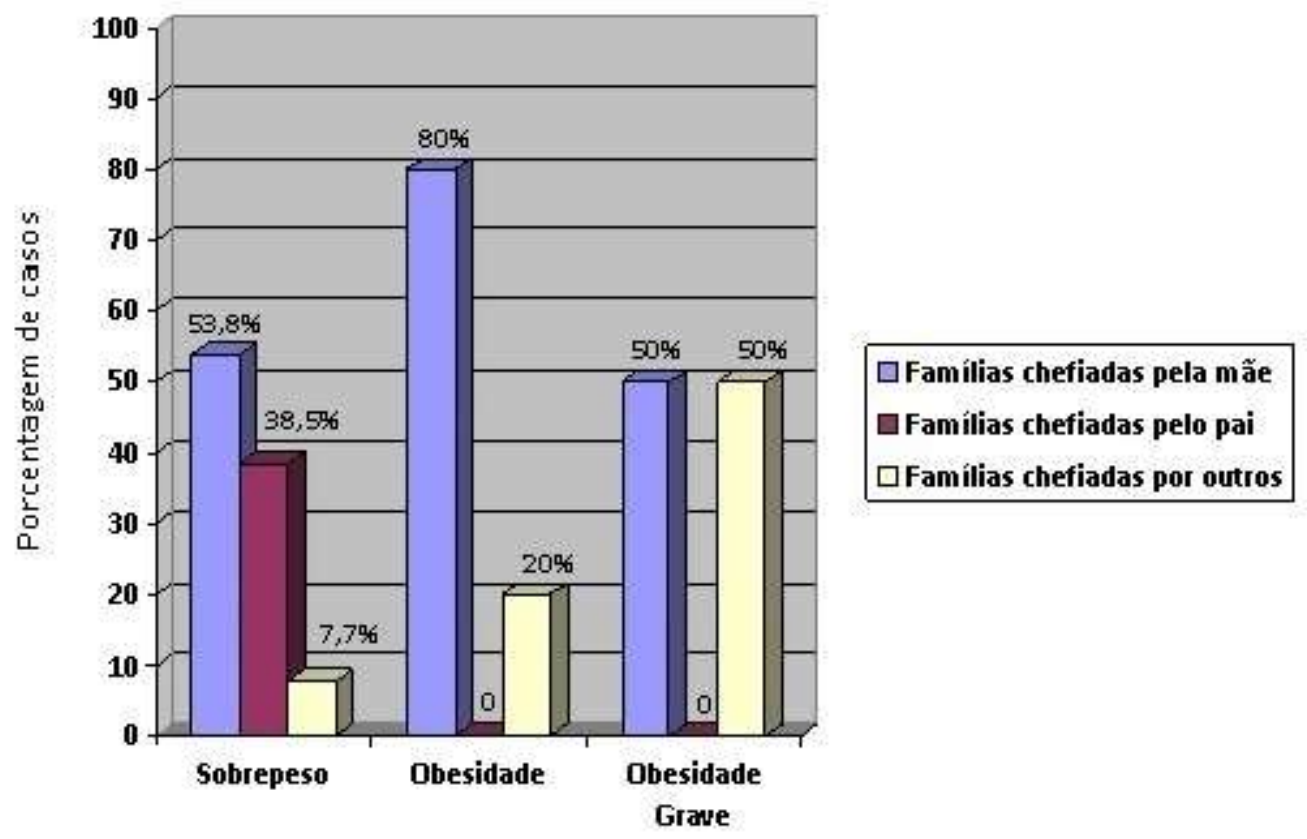

Estado nutricional dos alunos

Fonte: Autores.

O baixo nível de escolaridade das mães, no entanto, não aparece nesta pesquisa como fator preponderante quanto aos diagnósticos de sobrepeso, obesidade e obesidade grave entre os adolescentes, visto que, entre as mães dos alunos com perfil nutricional eutrófico/desejável foi detectada, também, carência de formação, sendo que 54,3\% delas não chegaram a concluir nem mesmo o ensino fundamental, 4,3\% concluíram apenas essa etapa dos estudos, 6,5\% não concluíram o ensino médio, 19,6\% foram apontadas com ensino médio completo, $4,4 \%$ finalizaram o ensino superior e 10,9\% ficaram sem resposta. Entre as mães dos estudantes diagnosticados com sobrepeso, 38,5\% não chegaram a concluir o ensino fundamental, 7,7\% concluíram essa etapa dos estudos, $23 \%$ não encerraram o ensino médio e 30,8\% apareceram com ensino médio completo. Nenhuma delas chegou a acessar o ensino superior. Quando se analisa as mães dos alunos obesos, $60 \%$ apareceram com ensino fundamental incompleto, $20 \%$ com ensino médio também incompleto e $20 \%$ com o ensino médio finalizado. Da mesma forma, nenhuma delas chegou ao nível de graduação. Quanto às mães dos alunos enquadrados no estado de obesidade grave, observou-se que metade não finalizou nem mesmo o ensino fundamental, mas a outra metade, 50\%, concluiu o ensino superior (Figura 5). 
Figura 5: Relação estado nutricional de escolares adolescentes x Escolaridade da mãe no município de Fortaleza, CE.

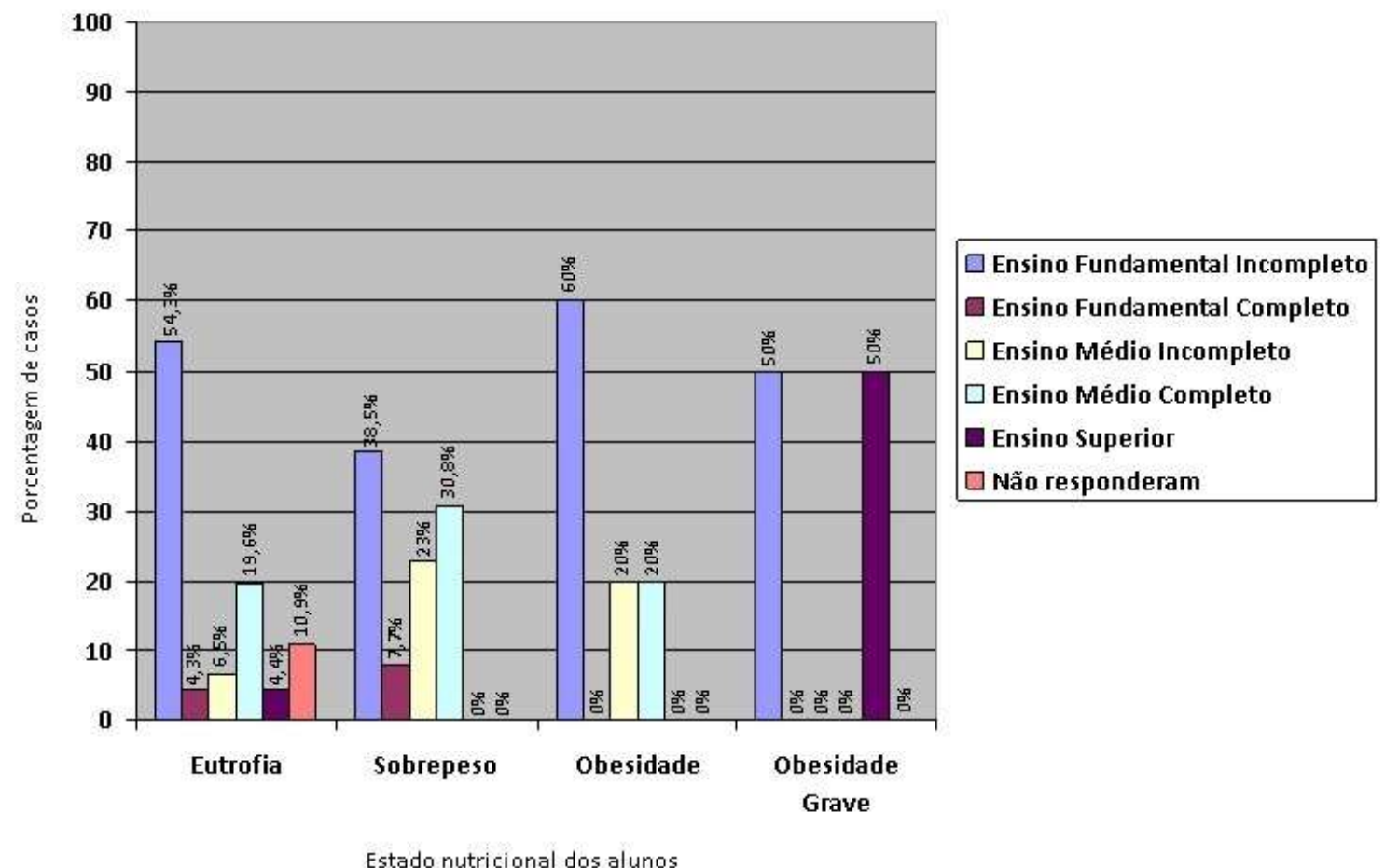

Fonte: Autores.

Contudo, Giugliano e Carneiro (2004) apontam, a partir de pesquisa realizada com 452 escolares, que é mais frequente a baixa escolaridade do que o ensino superior entre as mães dos alunos diagnosticados com sobrepeso e obesidade, havendo uma correlação inversa entre o desempenho escolar materno e a ocorrência desses quadros nutricionais entre seus filhos. Ainda segundo os pesquisadores, essa relação de inversibilidade não é percebida quando relacionados os casos de sobrepeso e obesidade com a escolaridade do pai. No entanto, como afirma Cerqueira (2020), a baixa escolaridade dos pais e das mães dificulta a superação dos altos índices de sobrepeso e de obesidade entre adolescentes, ao passo que a mudança comportamental necessária para resolução desse problema começa no ambiente familiar. Tenório e Cobayashi (2011) destacam, ainda, que a falta de formação de pais e mães atravanca a aceitação e a assimilação dos discursos de especialistas sobre a obesidade, bem como a compreensão das tabelas de crescimento, como as da OMS, apresentadas nesta pesquisa.

\subsection{A ausência da mulher no ambiente doméstico e seus impactos sobre os hábitos alimentares de escolares adolescentes}

Quando analisada a inclusão ou exclusão de refeições dos adolescentes, relacionando com a presença da mulher no ambiente domiciliar, verificou-se que, entre os escolares com famílias chefiadas pela mãe, ou seja, que as mães trabalham fora de casa, 44,1\% consomem café da manhã todos os dias, enquanto que nas famílias em que a mãe permanece no ambiente doméstico esse percentual sobe para 54,5\%. Os consumos diários de almoço e jantar também são maiores entre os alunos que contam com a presença materna ao longo do dia (78,8\% e 51,5\%, respectivamente), do que entre os que possuem as mães engajadas no mercado de trabalho (64,7\% e 50\%, respectivamente) (Figura 6). Os demais trocam o almoço e/ou jantar por lanches ao menos uma vez por semana. 
Figura 6: Realização diária de refeições por escolares adolescentes x Presença/ausência da mãe na rotina doméstica no município de Fortaleza, CE.

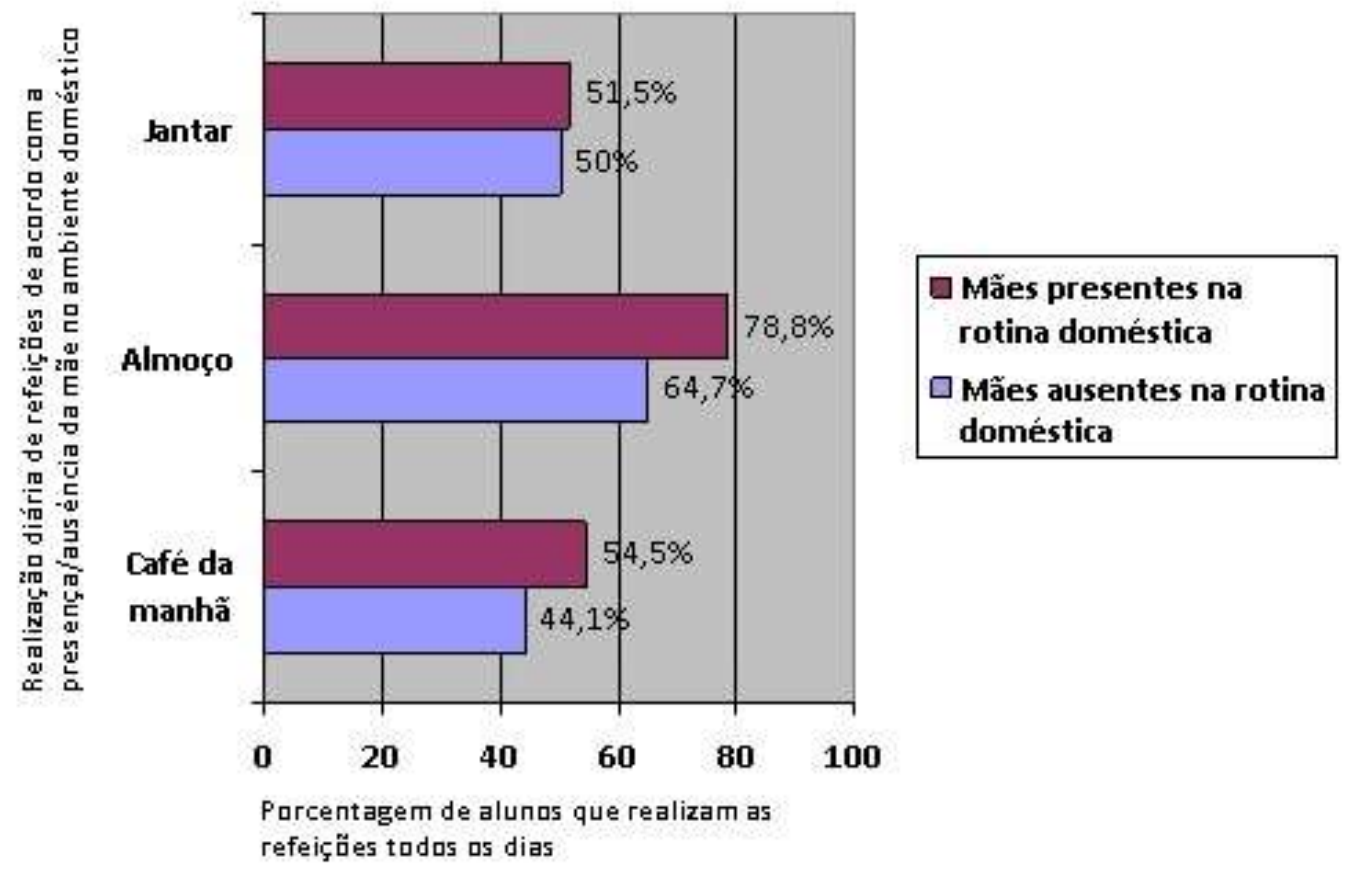

Fonte: Autores.

No entanto, a busca por fast-foods chega a ser maior entre os estudantes que têm a mãe presente na rotina doméstica, sendo que: $51,5 \%$ consomem esse tipo de comida de uma a duas vezes por semana; $27,3 \%$, de três a quatro vezes por semana; $6 \%$, de cinco a mais vezes por semana; e 15,2\%, nunca ou quase nunca. Entre os alunos com a mãe em plena atividade laboral, $47,1 \%$ tem acesso a fast-foods de uma a duas vezes por semana; $14,7 \%$, de três a quatro vezes; $8,8 \%$, de cinco a mais vezes por semana; e 29,4\%, nunca ou quase nunca (Figura 7). 
Figura 7: Consumo de fast-foods por escolares adolescentes x Presença/ausência da mãe na rotina doméstica no município de Fortaleza, CE

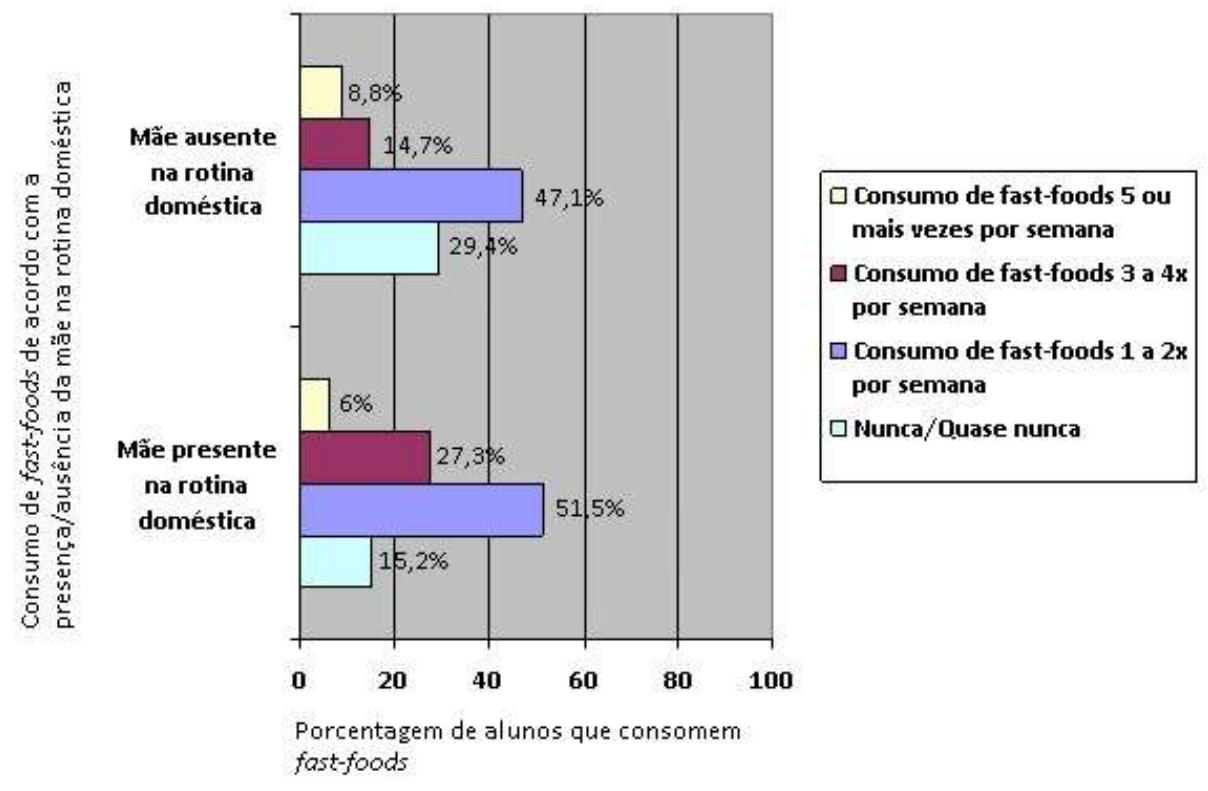

Fonte: Autores.

Visto isso, é possível considerar que a presença da mãe na rotina doméstica contribui para o ordenamento das refeições diárias dos adolescentes, principalmente o café da manhã, porém não minimiza o consumo de alimentos fora do lar, o que pode ser justificado, sobretudo, pelas diferenças salariais entre homens e mulheres ainda impostas pelo mercado de trabalho. O acesso maior a fast-foods entre os alunos com pais chefiando a família e as mães dentro de casa talvez esteja ligado ao maior poder aquisitivo e, consequentemente, ao maior poder de compra, já que, de acordo com pesquisa divulgada pelo Instituto Brasileiro de Geografia e Estatística (IBGE) (2021), as mulheres receberam, em 2019, apenas 77,7\% do rendimento dos homens. Mas é preciso destacar que, do ponto de vista nutricional, o acesso a fast-foods é considerado algo extremamente negativo. Esses hábitos alimentares, intensificados pela correria do dia a dia, contribuem para o aumento do consumo de gorduras saturadas e trans, açúcares etc, assim como para a redução do consumo de produtos in natura, como carboidratos complexos, frutas, legumes, verduras e hortaliças. Isso acaba gerando um quadro de excesso calórico pela ingestão desenfreada de macronutrientes e uma deficiência de micronutrientes (vitaminas e sais minerais), cenário perfeito para o desenvolvimento de sobrepeso, obesidade e doenças crônicas não-transmissíveis, como diabetes, hipertensão arterial e vários tipos de câncer (Souza, 2010).

Quanto ao consumo de produtos in natura, como frutas e verduras, há uma pequena diferença entre os alunos que têm suas mães em casa durante o dia e os que não as têm. Nas famílias chefiadas pela mulher, em que esta não está presente na rotina doméstica, 44,1\% dos adolescentes comem frutas e 41,2\% comem verduras pelo menos cinco vezes por semana. Nas casas chefiadas pelos pais, em que a mãe integra o dia a dia dos adolescentes, $51,5 \%$ dos avaliados comem frutas e $39,4 \%$ comem verduras com essa mesma frequência (Figura 8). 
Figura 8: Consumo de frutas e verduras por escolares adolescentes x Inserção da mulher no mercado de trabalho no município de Fortaleza, CE.

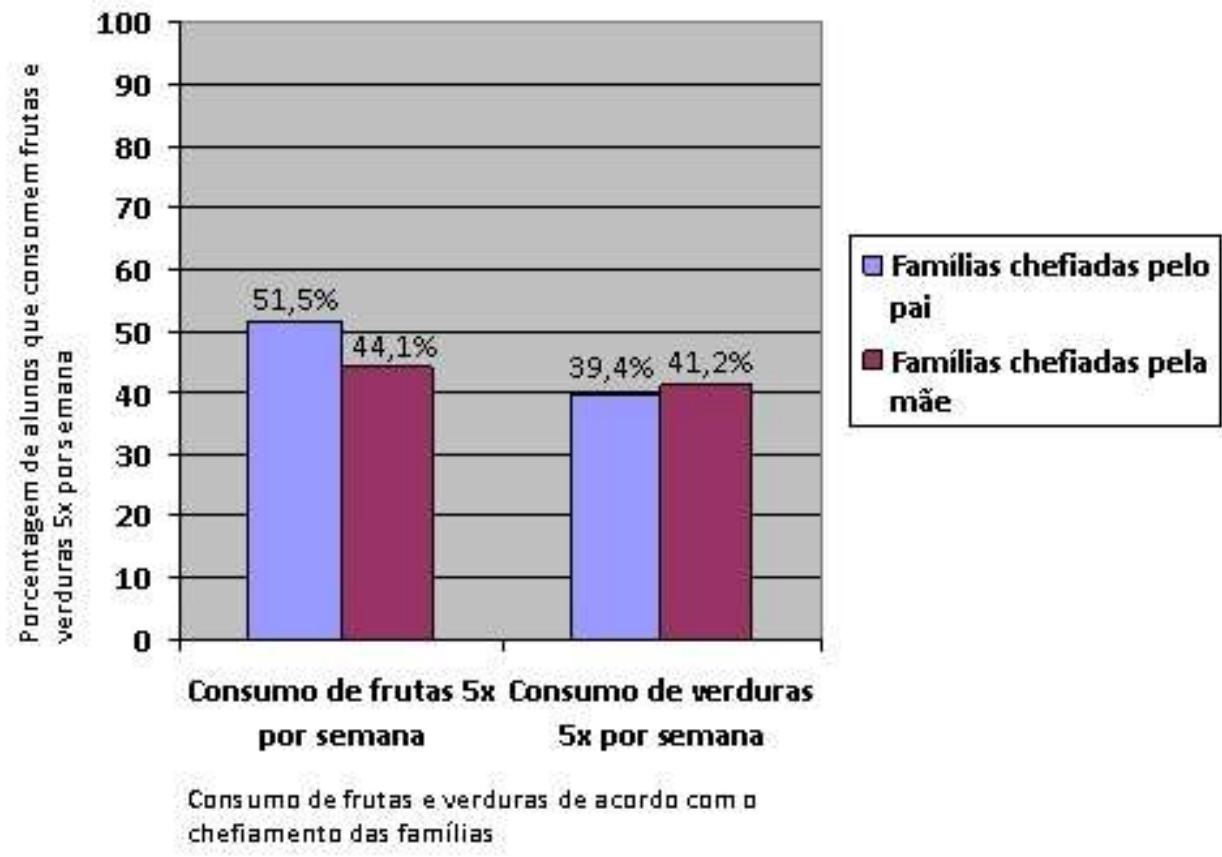

Fonte: Autores.

Essa pequena diferença traz uma reflexão sobre a resistência que adolescentes ainda impõem ao consumo desses alimentos, bem como sobre a necessidade de implementação de ações de Educação Alimentar e Nutricional (EAN) para promover práticas alimentares saudáveis por meio da formação do conhecimento e do desenvolvimento da autonomia e criticidade do adolescente, viabilizando a reavaliação de seus hábitos alimentares e a tomada de decisão no sentido de alterar quadros nutricionais compreendidos como inadequados. Nesse contexto, Viana et al. (2020) destacam a importância do binômio educação/nutrição como estratégia efetiva de combate ao sobrepeso e à obesidade, em paralelo ao binômio renda/nutrição e à realização frequente de inquéritos nutricionais no ambiente escolar. Ou seja, além de garantir recursos financeiros suficientes para acesso a uma alimentação adequada e saudável, é indispensável educar.

As escolas de tempo integral são espaços promissores para a implementação dessas medidas, como relatam Rabelo et al. (2020) a partir de uma experiência extensionista nas escolas de ensino médio de tempo integral do estado do Ceará. Essas instituições têm como um de seus princípios o estímulo a autonomia e ao protagonismo dos alunos, sendo a oferta de disciplinas eletivas uma das táticas utilizadas para o desenvolvimento de habilidades que ultrapassam a instrumentalização viabilizada pelo chamado currículo básico. Na experiência narrada, os pesquisadores destacam a elaboração de um programa de Educação Alimentar e Nutricional para a disciplina de Segurança Alimentar e Nutricional posto em prática ao longo dos três anos de vigência do projeto de extensão do curso de Gastronomia da Universidade Federal do Ceará, contemplando 300 estudantes de cinco escolas da rede estadual de ensino, que optaram por acompanhar as referidas aulas voluntariamente, demonstrado interesse na temática.

De acordo com o Marco de Referência de Educação Alimentar e Nutricional para as Políticas Públicas, o conceito de EAN se consolida a partir do Direito Humano à Alimentação Adequada e da garantia da Segurança Alimentar e Nutricional, consistindo em "um campo de conhecimento e de prática contínua e permanente, transdisciplinar, intersetorial e multiprofissional que visa promover a prática autônoma e voluntária de hábitos alimentares saudáveis” (Brasil, 2012, p. 23). 
Silva et al. (2012), por sua vez, destacam que a alimentação e a nutrição adequadas constituem requisito para o desenvolvimento e o crescimento com qualidade de vida, prevenindo doenças carenciais ou a obesidade e comorbidades associadas a ela. Além disso, representa um direito humano, estando, hoje, a educação nutricional intimamente ligada à promoção da saúde, de acordo com as diretrizes da Política Nacional de Alimentação e Nutrição do Ministério da Saúde. Para tanto, Bezerra (2018, p. 29) aponta o Guia Alimentar para a População Brasileira como "um instrumento pedagógico por excelência, indispensável à prática dos profissionais que atuam em áreas e setores que desenvolvem atividades de EAN". Desta forma, recomenda-se a aplicação e o uso do Guia Alimentar para a População Brasileira no ambiente escolar.

\section{Considerações Finais}

Pondera-se, portanto, com o presente estudo, que há uma considerável relação entre os diagnósticos de sobrepeso, obesidade e obesidade grave entre adolescentes escolares com o fato de suas mães estarem inseridas no mercado de trabalho. Foi possível observar, ainda, que a presença feminina no ambiente domiciliar contribuiu para o ordenamento das refeições, no entanto, não foi fator definidor dos hábitos alimentares do grupo avaliado. Nota-se, inclusive, um maior consumo de fast-foods entre as famílias chefiadas pela figura masculina e em que a mãe se faz presente no dia a dia, o que nos faz questionar se isso tem ligação com os maiores salários ainda atribuídos aos homens. Contudo, mesmo chegando ao diagnóstico de que os alunos que convivem com suas mães em casa durante todo o dia consomem mais fast-foods, pode-se considerar que a mulher continua assumindo o papel de cuidado e controle sobre a alimentação das famílias brasileiras, já que foram encontrados menos casos de sobrepeso e obesidade entre os avaliados que contam com a presença de suas mães na rotina de suas casas.

Faz-se, então, urgente uma intervenção por meio das políticas públicas voltadas às mulheres, principalmente às chefes de famílias brasileiras, no sentido de dar suporte àquelas que acumulam suas atividades laborais e as obrigações domésticas. A crescente inserção feminina no mercado de trabalho requer uma reorganização social, tanto em relação à equiparação de salários com os homens, para que estas possam comprar alimentos e alimentar adequadamente seus filhos, como no suprimento das carências que se formam no âmbito familiar a partir de sua ausência, sendo a Educação Alimentar e Nutricional, à toda família, uma delas. Assim, as escolas devem cumprir o papel de formadoras e desenvolvedoras do senso crítico de crianças e adolescentes acerca da importância da manutenção de hábitos alimentares saudáveis, bem como, promover sua autonomia alimentar.

Dessa forma, surgem, aqui, novos caminhos para estudos e pesquisas voltadas à avaliação das políticas públicas implementadas pelo Estado brasileiro que busquem a diminuição das desigualdades de gênero no país e que impactem, diretamente, no acesso a recursos financeiros que viabilizem uma alimentação adequada e saudável à todos; à análise das potencialidades do Guia Alimentar para a População Brasileira como ferramenta de Educação Alimentar e Nutricional nas escolas de tempo integral; e à importância da realização de inquéritos nutricionais nas escolas brasileiras para redução dos índices de sobrepeso e obesidade entre crianças e adolescentes.

\section{Referências}

Andrade, M. M. de (2010). Introdução à metodologia do trabalho científico. Atlas.

Batista Filho, M. \& Rissin, A. (2003). A transição nutricional no Brasil: tendências regionais e temporais. Cad. Saúde Pública, 19 (1), $181-191$.

Brasil (2014). Guia alimentar para a população brasileira (2a ed.). Ministério da Saúde, Secretaria de Atenção à Saúde, Departamento de Atenção Básica.

Brasil (2012). Marco de referência de educação alimentar e nutricional para as políticas públicas. Ministério do Desenvolvimento Social e Combate à Fome, Secretaria Nacional de Segurança Alimentar e Nutricional.

Brasil (2020). Pesquisa Nacional de Saúde 2019: Atenção primária à saúde e informações antropométricas. IBGE, Coordenação de Trabalho e Rendimento. Brasil (2016a). Pesquisa Nacional de Saúde do Escolar 2015. IBGE, Coordenação de População e Indicadores Sociais. 
Research, Society and Development, v. 10, n. 7, e25010716586, 2021

(CC BY 4.0) | ISSN 2525-3409 | DOI: http://dx.doi.org/10.33448/rsd-v10i7.16586

Brasil (2016b). Relatório do Estado Nutricional de adolescentes no Brasil. Ministério da Saúde, Sistema de Vigilância Alimentar e Nutricional - SISVAN.

Bezerra, J. A. B. (2018). Educação Alimentar e Nutricional: articulação de saberes. Edições UFC.

Cerqueira, R. A. (2020). Estratégias nutricionais para a prevenção e tratamento da obesidade na infância e adolescência. Monografia de Bacharelado em Nutrição. Faculdade de Ciências da Educação e Saúde, Centro Universitário de Brasília.

Giugliano, R. \& Carneiro, E. C. (2004). Fatores associados à obesidade em escolares. Jornal de Pediatria. 80 (1), 17-22.

Guimarães, T. A. de S. F.; França, L. P. S. \& Oliveira, L. M. N. de (2020). Perfil nutricional e glicemia de adolescentes escolares. Research, Society and Development, 9 (3), 1-16.

IBGE (2021). Estatísticas de Gênero: Indicadores Sociais das Mulheres no Brasil (2a ed.). IBGE.

LANPOP (2020). Perguntas frequentes sobre antropometria e avaliação de estado nutricional. http://www.fsp.usp.br/lanpop/faq/.

Lelis, C. T.; Teixeira, C. M. D. \& Silva, N. M. (2012). A inserção feminina no mercado de trabalho e suas implicações para os hábitos alimentares da mulher e de sua família. Revista Saúde em Debate. 36 (95), 523-532.

Lüdke, M. \& André, M. E. D. A. (1986). Pesquisa em educação: abordagens qualitativas. E.P.U.

Ministério da Saúde (2019). Curvas de Crescimento da Organização Mundial da Saúde - OMS. https://aps.saude.gov.br/ape/vigilanciaalimentar/curvascrescimento.

Pereira, A. S.; Shitsuka, D. M.; Parreira, F. J. \& Shitsuka, R. (2018). Metodologia da pesquisa científica. UFSM.

Rabelo, C. A. F.; Mendes, A. E. P.; Holanda, L. E. O.; Souza, F. E. B.; Lima, D. da S.; Amaral, R. Q. G. do; Bezerra, J. A. B. \& Sousa, P. H. M. de (2020). Promoção da segurança alimentar e nutricional em escolas de ensino médio em tempo integral: relato de experiência extensionista. Research, Society and Development, 9 (12), 1-21.

Rodrigues, P. R. M (2013). Hábitos alimentares, estilo de vida e estado nutricional de adolescentes: um estudo de base escolar em Cuiabá/MT. Tese de doutorado em nutrição. Instituto de Nutrição, Universidade Federal do Rio de Janeiro.

Santos, D. S. dos; Carneiro, M. de S.; Silva, S. C. M. e; Aires, C. N.; Carvalho, L. J. da S. \& Costa, L. C. B. (2019). Transição nutricional na adolescência: uma abordagem dos últimos 10 anos. Revista Eletrônica Acervo Saúde, v. 20, 477-481.

Silva, J. G. da; Teixeira, M. L. de O. \& Ferreira, M. de A. (2012). Alimentação e saúde: sentidos atribuídos por adolescentes. Escola Anna Nery. 16 (1), p. 88 95 .

SISVAN (2019). Nota Técnica SISVAN Municipal - Estado Nutricional dos Usuários da Atenção Básica. http://tabnet.datasus.gov.br/cgiwin/SISVAN/CNV/notas_sisvan.html\#.

Souza, E. B. de (2010). Transição nutricional no Brasil: analise dos principais fatores. Caderno UNIFOA, n.13, 49-53.

Tenorio, A. e S. \& Cobayashi, F. (2011). Obesidade infantil na percepção dos pais. Revista Paulista de Pediatria, 29 (4), 634-639.

Viana, R.; Fumagalli, L. M. R. \& Ilha, P. V. (2020). Estado nutricional e fatores relacionados de escolares. Research, Society and Development, 9 (1), 1-14.

World Health Organization - WHO (2007). Growht reference data for 5-19 years. https://www.who.int/tools/growth-reference-data-for-5to19-years 